\title{
Prolonged exposure of mouse and human podocytes to insulin induces insulin resistance through lysosomal and proteasomal degradation of the insulin receptor
}

\author{
Abigail C. Lay ${ }^{1}$ - Jenny A. Hurcombe ${ }^{1}$ - Virginie M. S. Betin ${ }^{1} \cdot$ Fern Barrington ${ }^{1}$.

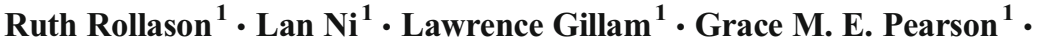 \\ Mette V. Østergaard ${ }^{1,2}$ - Hellyeh Hamidi ${ }^{3}$. Rachel Lennon ${ }^{3}$ - Gavin I. Welsh ${ }^{1}$. \\ Richard J. M. Coward ${ }^{1}$
}

Received: 21 February 2017 / Accepted: 28 June 2017 /Published online: 29 August 2017

(C) The Author(s) 2017. This article is an open access publication

\begin{abstract}
Aims/hypothesis Podocytes are insulin-responsive cells of the glomerular filtration barrier and are key in preventing albuminuria, a hallmark feature of diabetic nephropathy. While there is evidence that a loss of insulin signalling to podocytes is detrimental, the molecular mechanisms underpinning the development of podocyte insulin resistance in diabetes remain unclear. Thus, we aimed to further investigate podocyte insulin responses early in the context of diabetic nephropathy.

Methods Conditionally immortalised human and mouse podocyte cell lines and glomeruli isolated from $d b / d b$ DBA/ $2 \mathrm{~J}$ mice were studied. Podocyte insulin responses were investigated with western blotting, cellular glucose uptake assays and automated fluorescent imaging of the actin cytoskeleton. Quantitative (q)RT-PCR was employed to investigate changes in mRNA. Human cell lines stably overproducing the insulin receptor (IR) and nephrin were also generated, using lentiviral constructs.

Results Podocytes exposed to a diabetic environment (high glucose, high insulin and the proinflammatory cytokines TNF- $\alpha$

Electronic supplementary material The online version of this article (doi:10.1007/s00125-017-4394-0) contains peer-reviewed but unedited supplementary material, which is available to authorised users.
\end{abstract}

Richard J. M. Coward

richard.coward@bristol.ac.uk

1 Bristol Renal, Bristol Medical School, University of Bristol, Whitson Street, Bristol BS1 3NY, UK

2 Global Research, Novo Nordisk A/S, Måløv, Denmark

3 Wellcome Trust Centre for Cell-Matrix Research, Division of Cell Matrix Biology and Regenerative Medicine, School of Biology, Faculty of Biology, Medicine and Health, Manchester Academic Health Science Centre, University of Manchester, Manchester, UK and IL-6) become insulin resistant with respect to glucose uptake and activation of phosphoinositide 3-kinase (PI3K) and mitogen-activated protein kinase (MAPK) signalling. These podocytes lose expression of the IR as a direct consequence of prolonged exposure to high insulin concentrations, which causes an increase in IR protein degradation via a proteasomedependent and bafilomycin-sensitive pathway. Reintroducing the IR into insulin-resistant human podocytes rescues upstream phosphorylation events, but not glucose uptake. Stable expression of nephrin is also required for the insulin-stimulated glucose uptake response in podocytes and for efficient insulin-stimulated remodelling of the actin cytoskeleton.

Conclusions/interpretation Together, these results suggest that IR degradation, caused by high levels of insulin, drives early podocyte insulin resistance, and that both the IR and nephrin are required for full insulin sensitivity of this cell. This could be highly relevant for the development of nephropathy in individuals with type 2 diabetes, who are commonly hyperinsulinaemic in the early phases of their disease.

Keywords Albuminuria - Diabetic nephropathy · Genetic background $\cdot$ Insulin resistance $\cdot$ Kidney injury

$\begin{array}{ll}\text { Abbreviations } & \\ \text { IR } & \text { Insulin receptor } \\ \text { MAPK } & \text { Mitogen-activated protein kinase } \\ \text { mTOR } & \text { Mechanistic target of rapamycin } \\ \text { qRT-PCR } & \text { Quantitative RT-PCR } \\ \text { ROS } & \text { Reactive oxygen species } \\ \text { WT } & \text { Wild type } \\ \text { WT-IR-Neph } & \text { Wild-type cells producing insulin } \\ & \text { receptor and nephrin }\end{array}$


WT-Neph Wild-type cells producing nephrin

VEGF Vascular endothelial growth factor

\section{Introduction}

Diabetic nephropathy, in which albuminuria is an early manifestation, occurs in approximately one third of diabetic individuals and is the leading cause of end-stage renal failure worldwide [1]. It is well recognised that insulin resistance plays a major role in the pathogenesis of both type 1 and type 2 diabetes [2,3], including a role in the development of renal complications. Interestingly, in addition to being associated with albuminuria and nephropathy in both type $1[4,5]$ and type 2 [6] diabetes, insulin resistance is also associated with the development of albuminuria in non-diabetic individuals [7]. Renal disease is also common among people with severe forms of genetic insulin resistance [8].

A range of circulating factors are dysregulated early in the development of systemic insulin resistance, as either a cause or a consequence of disrupted cellular insulin signalling. Among these factors, elevated inflammatory cytokines, hyperglycaemia and hyperinsulinaemia are particularly prominent [9]. Although the negative regulation of insulin signalling is relatively well characterised in classical insulin-responsive tissues, such as skeletal muscle and liver, the impairment of insulin action does not occur uniformly throughout all insulin-responsive tissues, and the effects of factors associated with insulin resistance are often tissue-specific [10].

Podocytes are specialised epithelial cells lining the urinary side of the glomerular filtration barrier in the kidney, essential in the maintenance of glomerular function. Podocyte loss or disturbance is linked to the development of albuminuria and occurs early in the progression of diabetic nephropathy [11-14].

We previously demonstrated that podocytes are insulinresponsive cells in vitro [15], and the specific deletion of the podocyte insulin receptor (IR) in vivo disrupts glomerular function, causing features reminiscent of diabetic nephropathy, independent of hyperglycaemia [16]. Furthermore, a podocyte-specific reduction in IR production has been found to exacerbate albuminuria in a mouse model of diabetic nephropathy [17], further highlighting the importance of podocyte IR signalling in disease.

There is also increasing evidence that podocyte insulin responses may be negatively regulated in situations of systemic insulin resistance $[18,19]$. In particular, the finding that podocytes isolated from $d b / d b$ mice display reduced insulin-stimulated Akt phosphorylation may suggest that circulating factors, associated with type 2 diabetes, have the capacity to disrupt podocyte insulin responses early in the course of glomerular disease [20]. Despite this, relatively little is still known about the specific factors that regulate podocyte insulin responses.
This study aimed to investigate how factors associated with systemic insulin resistance influence podocyte insulin signalling and, consequently, the development of renal disease in situations of insulin resistance, including diabetic nephropathy.

\section{Methods}

Animals All animal experiments and procedures were approved by the UK Home Office in accordance with the Animals (Scientific Procedures) Act 1986, and the Guide for the Care and Use of Laboratory Animals was followed during experiments. Heterozygous $d b / w t$ DBA/2J (D2.BKS(D)-Lepr $\left.r^{d b} / \mathrm{J}\right)$ mice were purchased from the Jackson Laboratory (Bar Harbor, ME, USA). Female and male $d b / d b$ mice were bred in house, as described [21]. Glomeruli were isolated after perfusion with dynabeads (Thermo Fisher, Paisley, UK). Further details are available in the electronic supplementary material (ESM).

\section{Generation of podocyte cell lines from $d b / d b$ and wild-type} mice Podocytes were isolated from perfused glomeruli from a male $d b / d b$ DBA/2J and male wild-type (WT) DBA/2J littermate control mouse at 12 weeks of age. These podocytes were conditionally immortalised with temperature-sensitive SV40 transfection as previously described [22, 23].

Cell culture Conditionally immortalised human [24] and mouse [22] podocytes were maintained in RPMI-1640 containing L-glutamine and $\mathrm{NaHCO}_{3}$, supplemented with $10 \%$ FBS (Sigma Aldrich, Gillingham, UK). Cells were studied after $12-14$ days differentiation at $37^{\circ} \mathrm{C}$ and were free of Mycoplasma infection.

Cell treatments To mimic a diabetic environment in vitro, podocytes were grown in the presence of $100 \mathrm{nmol} / \mathrm{l}$ insulin (Tocris, Bristol, UK), $25 \mathrm{mmol} / \mathrm{l}$ glucose (Sigma), $1 \mathrm{ng} / \mathrm{ml}$ TNF- $\alpha$ and $1 \mathrm{ng} / \mathrm{ml}$ IL-6 (R\&D systems, Abingdon, UK). DMannitol (Sigma) was used as a control for osmotic pressure in these assays. For initial chronic insulin exposure, podocytes were incubated with insulin at $10 \mathrm{nmol} / 1$ and $100 \mathrm{nmol} / \mathrm{l}$ for 10 days. Although supraphysiological (as physiological hyperinsulinaemia is typically within the range 1000 $2000 \mathrm{pmol} / \mathrm{l}$, occurring over an extended period of months or years), this is consistent with numerous in vitro studies of other cell types [25-30]. For short-term insulin stimulation, culture medium was replaced with serum- and insulin-free RPMI-1640 for 2-4 h, and podocytes were re-challenged with insulin at 10 or $100 \mathrm{nmol} / \mathrm{l}$ for $10 \mathrm{~min}$. For inhibition of proteasomal and lysosomal degradation, podocytes were treated with $10 \mu \mathrm{mol} / \mathrm{l} \mathrm{MG}-132$ (Sigma) or $50 \mathrm{nmol} / \mathrm{l}$ bafilomycin (Tocris), respectively, for $8 \mathrm{~h}$. 
Lentiviral transfection of podocytes Human IR (NM_000208.2) was subcloned into pLenti-TetCMV(IR)Rsv(RFP-Bsd) expression vector (Gentarget, San Diego, CA, USA). The human nephrin expression plasmid (pWPXLNephrin-FLAG) was a gift from R. Lennon (University of Manchester). Expression vectors were transfected into Lenti-X 293 T cells (Clontech/Takara Bio Europe SAS, Saint-Germainen-Laye, France), together with packaging vectors pMD.2G (Addgene no. 12259) and psPAX2 (Addgene no. 12260), both gifts from D. Trono (École polytechnique fédérale de Lausanne), as previously reported [31]. Lentiviral particles were purified from the cell supernatant fraction, and immortalised podocytes were transduced overnight in the presence of polybrene. IRcontaining podocytes were selected using blasticidin.

Glucose uptake assays Insulin-stimulated glucose uptake into podocytes was measured as previously described [15]. Briefly, cells were serum-starved before incubation with a modified KRP solution (see ESM Methods) for $15 \mathrm{~min}$ at $37^{\circ} \mathrm{C}$. After appropriate stimulation, $\left[{ }^{3} \mathrm{H}\right] 2$-deoxy-D-glucose (Perkin Elmer, Coventry, UK) was added at $37 \mathrm{kBq} / \mathrm{ml}$ for $5 \mathrm{~min}$. Solubilised cell suspensions were collected, and radioactivity was measured in dpm using a multi-purpose scintillator counter (Beckman Coulter, High Wycombe, UK). Each condition was performed in duplicate or triplicate.

Quantitative RT-PCR Total RNA was isolated using TRIzol Reagent (Invitrogen/Thermo Fisher), and cDNA was synthesised using a high-capacity RNA-cDNA kit (Applied Biosystems/Thermo Fisher). Quantitative (q)RT-PCR was performed using SYBR green (Sigma) in an Applied
Biosystems StepOnePlus system and mRNA normalised to $\beta$-actin. Primer sequences are listed in ESM Methods.

Western blotting and antibodies Total protein lysates were extracted using RIPA lysis buffer (Sigma), resolved on 7.5$10 \%$ SDS-polyacrylamide gels and blotted onto nitrocellulose membranes. Membranes were incubated in primary antibodies overnight at $4^{\circ} \mathrm{C}$, before washing and incubation with the appropriate HRP-conjugated secondary antibody (Sigma) at a 1:10,000 dilution. Immunoreactive bands were visualised using Clarity ECL Western Blotting Substrate (Bio-Rad, Hemel Hempstead, UK) on a GE AI600 imager and quantified using ImageJ (NIH, https://imagej.nih.gov/ij/). Primary antibodies are listed in ESM Methods.

Immunoprecipitation For immunoprecipitation studies, $5 \mu \mathrm{g}$ of anti-IR $\beta$ (C-19)-AC or Rabbit IgG-AC (Santa Cruz, Dallas, TX, USA) was added directly to cell lysates and incubated overnight at $4^{\circ} \mathrm{C}$, under constant rotation. Immune complexes were pelleted at $10,000 \mathrm{~g}$ for $30 \mathrm{~s}$ and washed in lysis buffer. Immune complexes were eluted at $70^{\circ} \mathrm{C}$ for $10 \mathrm{~min}$. Immunoprecipitation samples were resolved on $7.5 \%$ SDSPAGE gels before western blotting.

Semi-automated immunofluorescent imaging and analysis Cells were grown in 96-well plates (Greiner, Stonehouse, UK), stimulated as indicated before fixation and immunostaining. Image acquisition was automated using an IN Cell Analyzer (GE Healthcare, Amersham, UK) imaging platform, and quantification was performed using IN Cell Analyzer work station 3.5 software. Three technical replicates were performed within each experiment, with four fields of view per well, yielding data

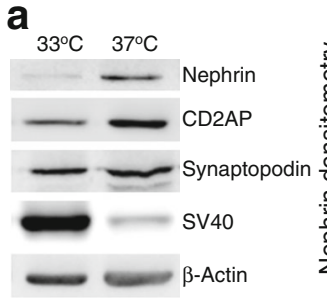

b

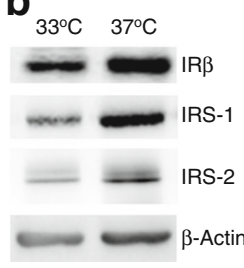

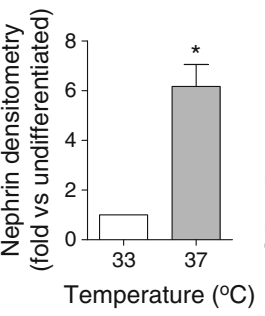
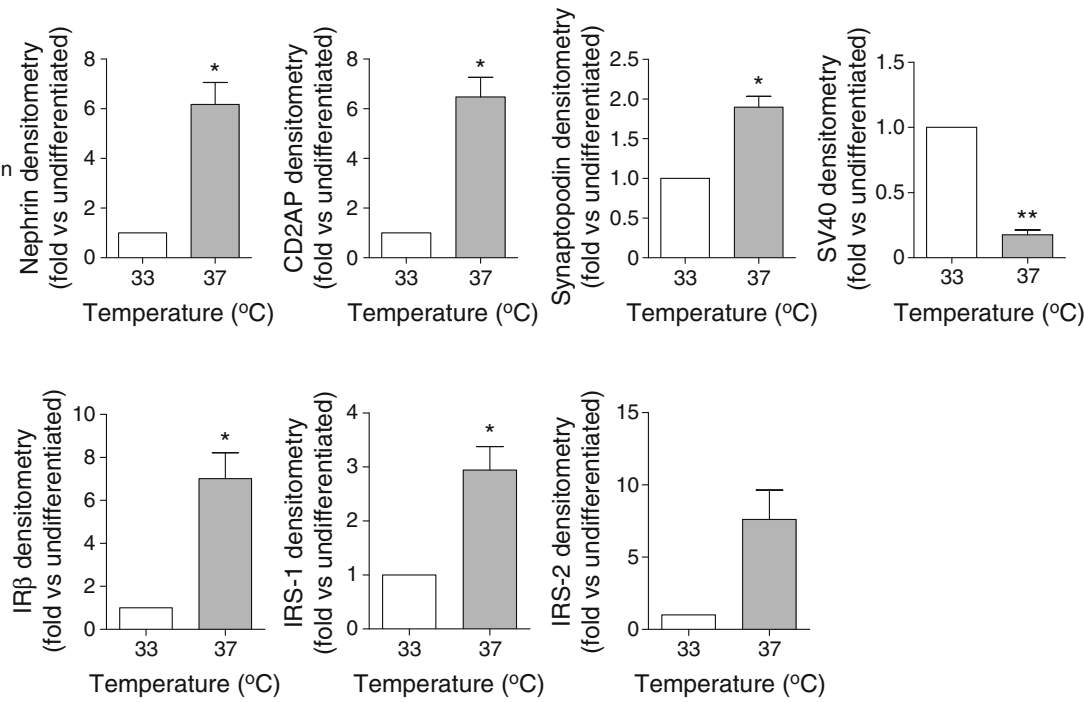

Fig. 1 Expression of podocyte markers and key insulin signalling proteins in WT mouse podocytes. Representative western blots and densitometry demonstrating (a) levels of the podocyte markers nephrin, CD2associated protein (CD2AP), synaptopodin and the heat-sensitive SV40 transgene, and (b) insulin signalling proteins IR $\beta$, IRS-1 and IRS-2, normalised to $\beta$-actin levels, under conditions of proliferation $\left(33^{\circ} \mathrm{C}\right)$ and differentiation $\left(37^{\circ} \mathrm{C}\right) . * p<0.05,{ }^{*} p<0.01$, unpaired $t$ test, $n=3$ 
for around 2000 cells per condition, per experiment. Additional details can be found in ESM Methods.

Statistical analysis Data are presented as means \pm SEM unless otherwise stated. Statistical analysis was performed using GraphPad Prism (GraphPad Software, La Jolla, CA, USA). Statistical significance was calculated with one-way ANOVA with Tukey's multiple comparison post hoc analysis, or unpaired two-tailed $t$ tests and taken as $p<0.05$.

\section{Results}

Culturing insulin-sensitive podocytes in a diabetic environment induces cellular insulin resistance Studying conditionally immortalised mouse podocytes [22], we initially demonstrated that, under basal conditions, these cells express key podocyte proteins and components of the insulin signalling cascade, which increase following podocyte differentiation (Fig. 1).

To determine whether these podocytes become insulin resistant following exposure to a diabetic environment in vitro,
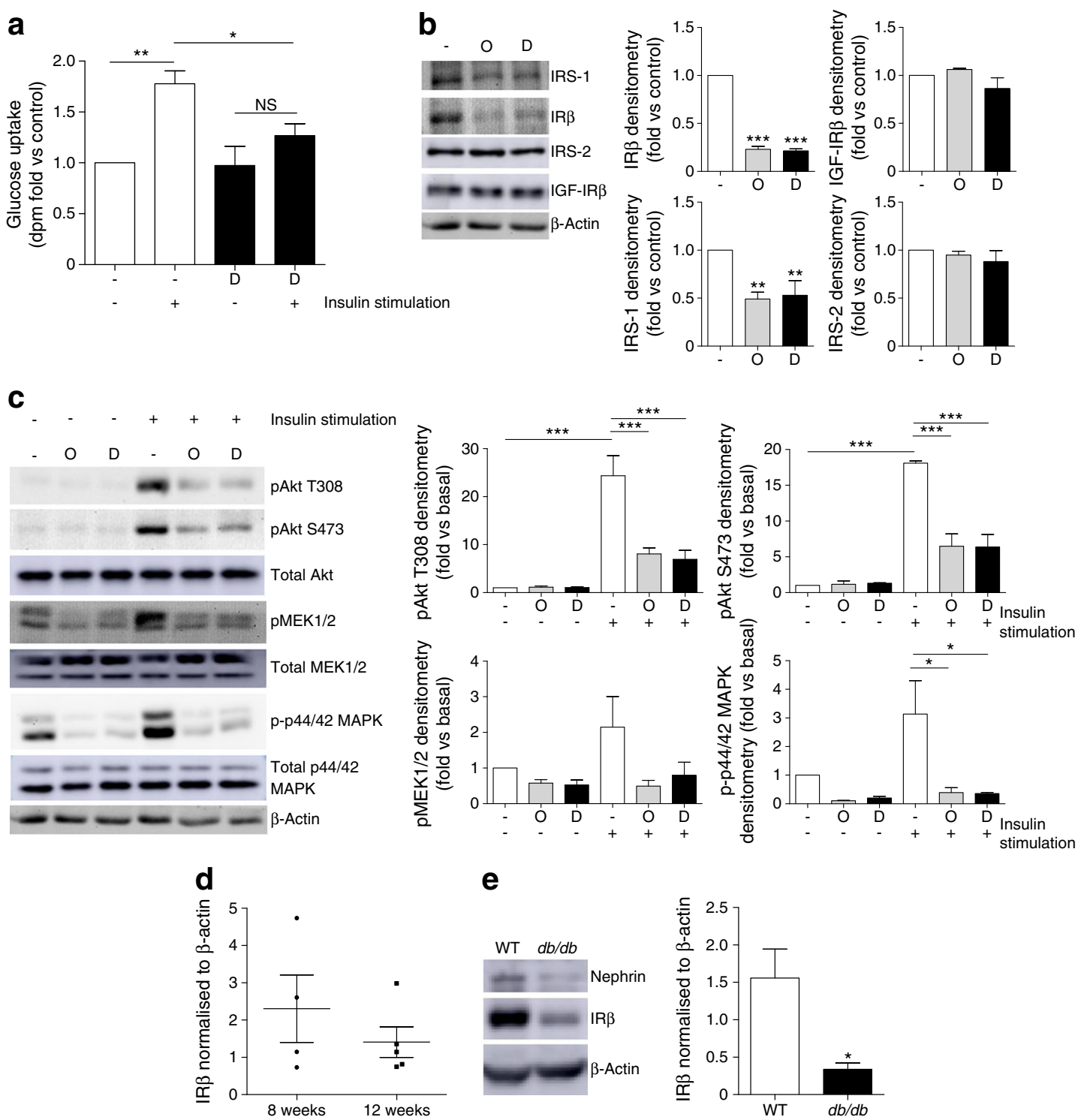

e

Fig. 2 Loss of IRs in diabetic mouse podocytes in vitro and in glomeruli from $d b / d b$ mice. WT mouse podocytes were treated for 10 days with $1 \mathrm{ng} / \mathrm{ml} \mathrm{TNF}-\alpha, 1 \mathrm{ng} / \mathrm{ml} \mathrm{IL}-6,100 \mathrm{nmol} / \mathrm{l}$ insulin and $25 \mathrm{mmol} / \mathrm{l}$ glucose (labelled Diabetic, D), prior to insulin stimulation (100 nmol/l). (a) dpm counts representing cellular uptake of $\left[{ }^{3} \mathrm{H}\right] 2$-deoxy-D-glucose following exposure of podocytes to the diabetic factors (D); $n=4$. (b) Representative western blots and densitometry of IRS-1, IR $\beta$, IRS-2 and IGF-IR $\beta$ protein following exposure of podocytes to the diabetic factors (D), or with mannitol (in parallel with insulin and inflammatory
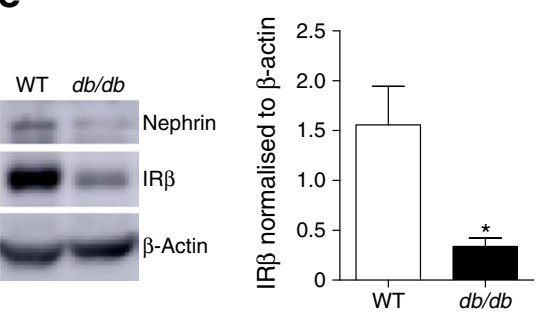

cytokines) included in place of glucose as an osmotic control $(\mathrm{O}) ; n=4$. (c) Representative western blots and densitometry of insulin-stimulated phosphorylation of Akt (T308, S473), mitogen-activated ERK-activating kinase (MEK1/2) and p44/42 MAPK (Thr202/Tyr204); $n=3$. (d) IR protein in glomeruli isolated from $d b / d b$ mice at $8(n=4$, two males and two females) and 12 weeks ( $n=5$, three males and two females) of age. (e) IR $\beta$ protein in podocyte cultures isolated from 3-month-old $d b / d b$ mice and WT littermate controls; $n=6$. $* p<0.05$, ** $p<0.001$, $* * * p<0.001$, one-way ANOVA, Tukey's multiple comparison 
cells were exposed to common factors associated with insulin resistance - inflammatory cytokines TNF- $\alpha$ and IL-6, high glucose and high insulin - for 10 days. Under these conditions, a significant reduction in insulin-stimulated glucose uptake was observed (Fig. 2a) and, interestingly, a significant reduction in podocyte IR and IRS-1 protein, although IGF-IR and IRS-2 were unchanged (Fig. 2b). These diabetic podocytes also showed a significant reduction in the insulin-stimulated phosphorylation of Akt (S473, T308) and p44/42 mitogenactivated protein kinase (MAPK) (Thr202/Tyr204) (Fig. 2c).

We have recently shown that $d b / d b$ mice on a DBA2/J background develop albuminuric renal disease in correlation with the level of systemic insulin resistance [21]. Glomeruli isolated from these mice demonstrated a trend towards a reduction in IR levels between 8 and 12 weeks of age (as diabetes and albuminuria progresses) (Fig. 2d). To determine podocyte IR production in this model, we isolated podocytes from male $d b / d b$ mice and male WT littermate control animals at 12 weeks of age and generated conditionally immortalised cell lines. Interestingly, podocytes isolated from $d b / d b$ animals had a significantly lower level of IR protein than podocytes isolated from WT littermate control mice (Fig. 2e). Primary podocytes isolated from $d b / d b$ mice also showed reduced IR production when compared with age- and sex-matched control mice (ESM Fig. 1).

Thus, factors associated with systemic insulin resistance modulate podocyte IR levels, attenuating insulin-stimulated phosphorylation cascades.

Chronic insulin exposure alone is responsible for podocyte IR loss and is sufficient to attenuate podocyte insulin signalling We next determined whether any individual factors were responsible for IR loss. Whereas chronic exposure to high glucose or TNF- $\alpha$ and IL-6 in isolation had no significant effect on podocyte IR levels (Fig. 3a, b), chronic exposure to insulin alone caused a significant reduction in podocyte IR protein, while IGF-IR, IRS-1 and IRS-2 were unchanged (Fig. 3c).

Consistent with reduced IR protein, we also found that chronic insulin exposure was sufficient to attenuate insulinstimulated phosphorylation of IR/IGF-IRs (using a phosphospecific antibody recognising both IR and IGF-IR) and Akt in podocytes. This was accompanied by a reduction in insulinstimulated glucose uptake (Fig. 4).
Fig. 3 Chronic insulin exposure is responsible for the loss of IR protein in mouse podocytes. Total IR $\beta$ levels following the exposure of podocytes to either (a) $25 \mathrm{mmol} / \mathrm{l}$ glucose $(\mathrm{G})$ or mannitol (M), or (b) TNF- $\alpha$ and IL- 6 , at the stated concentrations, for 10 days. Representative western blot (top) and matched densitometry (bottom). (c) Total IR $\beta$, IGF-IR $\beta$, IRS- 1 and IRS-2 protein following growth of podocytes in the presence of insulin in vitro. $* * * p<0.001$, one-way ANOVA, Tukey's multiple comparison; $n=4$ a
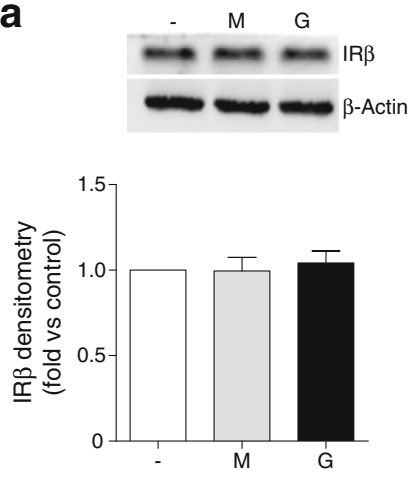

b
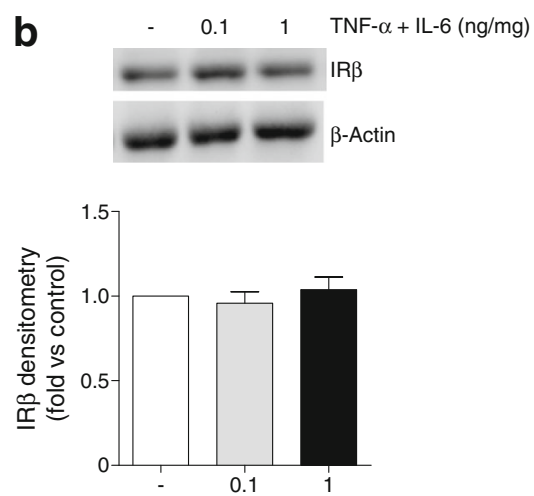

TNF $\alpha / \mathrm{lL}-6$ concentration ( $\mathrm{ng} / \mathrm{ml}$ )
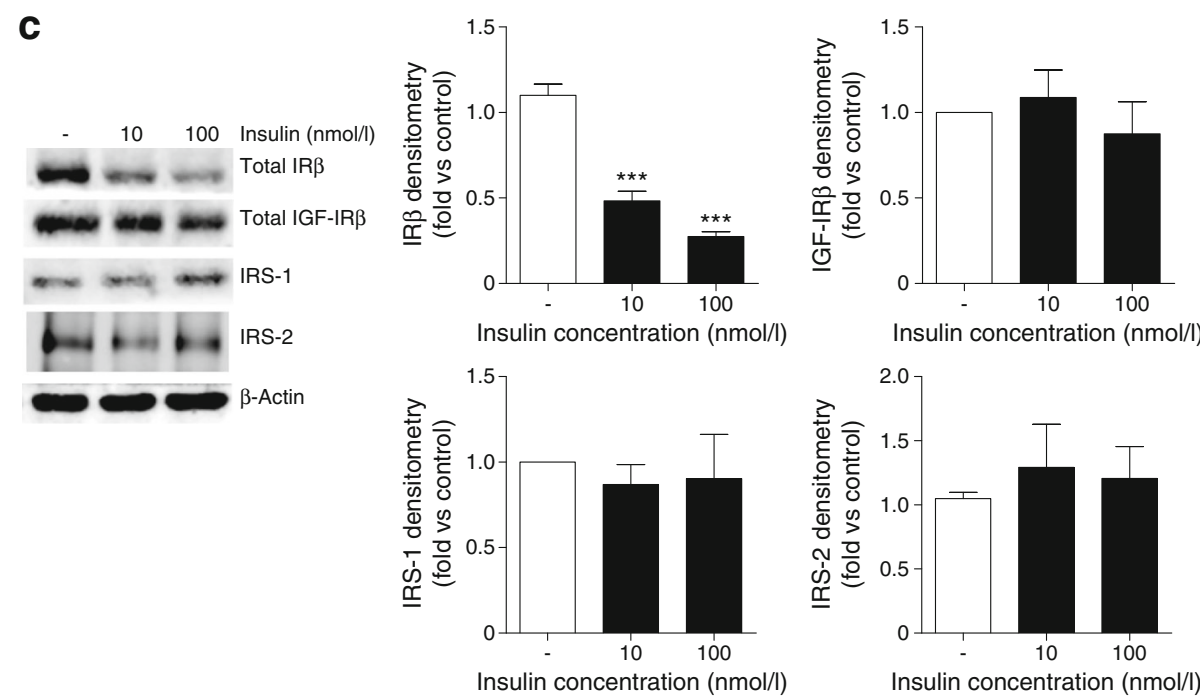

Insulin concentration (nmol/l) 
Fig. 4 Chronic insulin exposure is sufficient to attenuate podocyte insulin signalling. Mouse podocytes were grown in the presence of insulin (10 and $100 \mathrm{nmol} / \mathrm{l}$ ) prior to further insulin stimulation (black bars). (a) Representative western blots and matched densitometry of insulin-stimulated

phosphorylation cascades; $n=4$. $(\mathbf{b}, \mathbf{c})$ Insulin-stimulated glucose uptake assays; $n=3$ in duplicate. ${ }^{\dagger} p=0.041$, unpaired $t$ test; $* p<0.05, * * p<0.01$, $* * * p<0.001$, one-way ANOVA, Tukey's multiple comparison
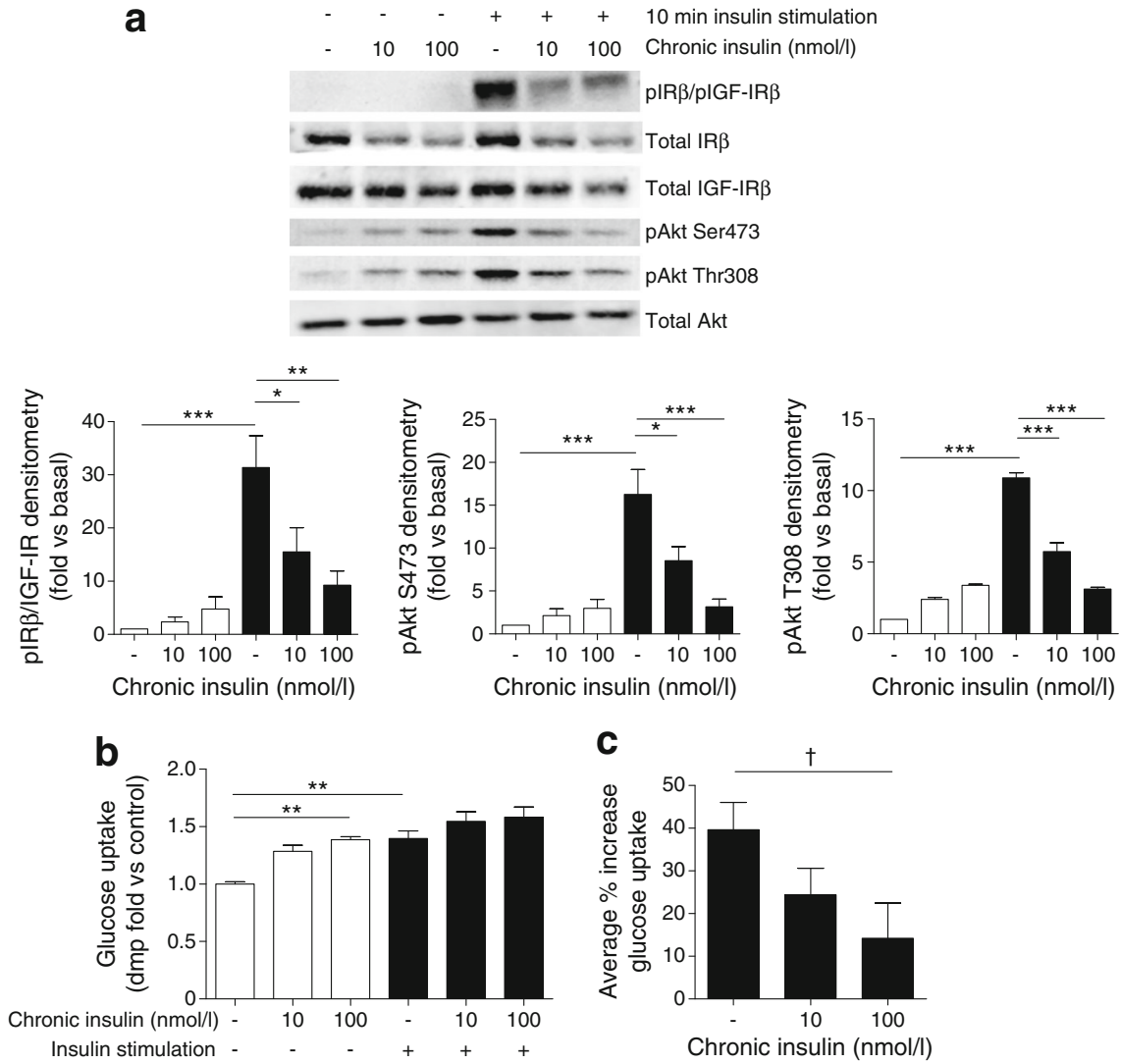

As podocyte IRS-1 was decreased in diabetic conditions (Fig. 2), we also examined the factors influencing IRS-1 levels. In contrast to the IR, chronic exposure to elevated glucose concentrations alone caused a significant reduction in podocyte IRS-1, as opposed to chronic insulin or inflammatory cytokine exposure (Fig. 5a). There was, however, no significant attenuation in insulin-stimulated phosphorylation cascades or glucose uptake under these conditions (Fig. 5b-d).
Fig. 5 High glucose is the cause of IRS-1 loss in podocytes.

Mouse podocytes were grown in the presence of $25 \mathrm{mmol} / 1$ glucose (G) or mannitol (M) for 10 days prior to insulin stimulation (100 nmol/1, $10 \mathrm{~min}$, black bars). (a) Representative western blot and densitometry of IRS-1 protein in podocytes. $n=3 ; * p<0.05$; ${ }^{\dagger} p=0.09$ vs control cells. (b) Representative western blots and densitometry of insulinstimulated signalling in podocytes following chronic glucose exposure; $n=3$. (c, d) Insulinstimulated glucose uptake assays $(n=4)$, in duplicate. ${ }^{*} p<0.05$, $* * p<0.01, * * * p<0.001$; no significant difference between any of the insulin-stimulated groups, one-way ANOVA, Tukey's multiple comparison a
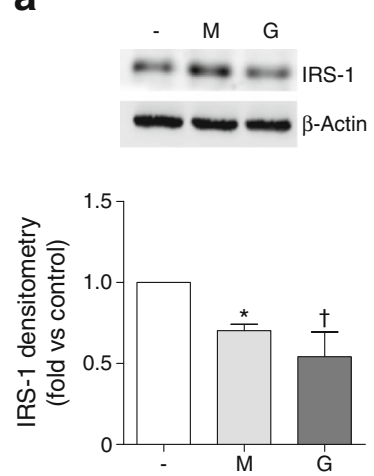

C

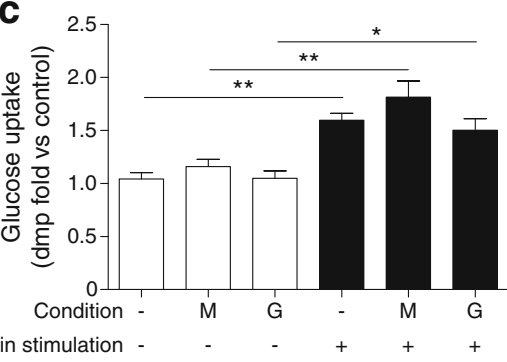

b
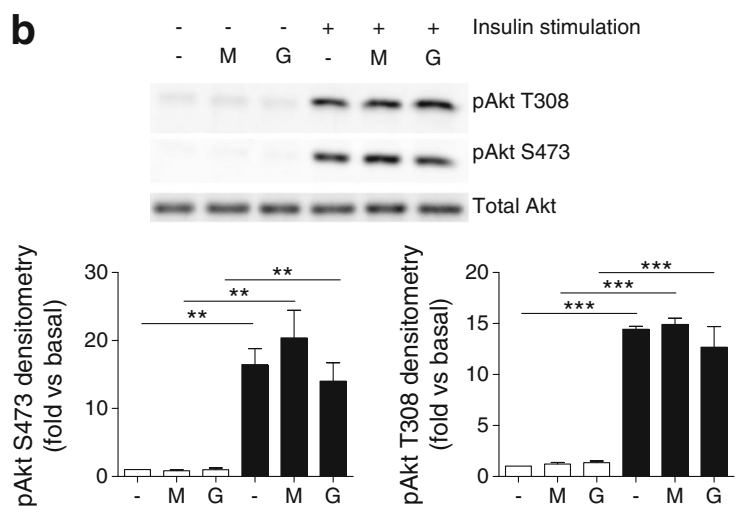

d

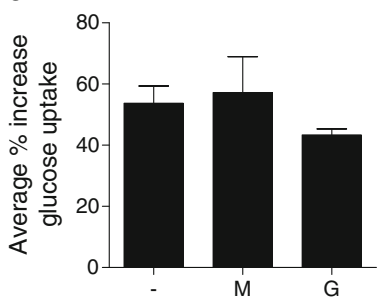


Increased IR degradation is responsible for insulininduced IR loss in podocytes To elucidate the mechanisms underlying IR loss in diabetic podocytes, we initially investigated whether insulin had any effect on IR transcription. Although insulin-induced inhibition of IR (also known as $I N S R$ ) mRNA has been reported in other cell systems [26, 27 , chronic insulin had no significant effect on mouse podocyte Ir mRNA levels (Fig. 6a, b).

The IR also exists as two isoforms as a consequence of the differential splicing of exon 11 [32], and the relative abundance of IR-A/B has been linked to hyperinsulinaemia [33]. As podocytes produce both IR isoforms [34], we quantified the relative abundance of $I r-A / B$ mRNA in podocytes using isoform-specific primers [35]. Although a significantly higher proportion of $I r-A$ mRNA (than that encoding $I r-B$ ) was observed in podocytes, there was no significant effect of chronic insulin exposure on the relative abundance of either isoform (Fig. 6c-f).

The role of post-translational degradation on insulininduced IR loss in podocytes was next examined. Consistent with the above results, chronic insulin stimulation caused a significant reduction in the levels of IR protein. Inhibition of lysosomal degradation in podocytes with bafilomycin (confirmed by a significant increase in p62, a protein typically degraded via autophagy and lysosomal pathways [36]) reduced this insulin-induced IR loss (Fig. 7a).

Inhibition of proteasomal degradation in podocytes by an $8 \mathrm{~h}$ incubation with the $26 \mathrm{~S}$ proteasome inhibitor MG132 (confirmed by an increase in ubiquitinated proteins) also delayed insulin-induced IR loss (Fig. 7b). In addition, we found evidence of IR ubiquitination. A small increase in ubiquitination was observed in IR $\beta$ immunoprecipitates where mouse podocytes were treated with both insulin and MG132. In mouse podocytes overproducing IR, we observed strong ubiquitin signals, confirming these results (ESM Fig. 2).

We saw no additional effect where proteasome and lysosome inhibitors were used in combination (Fig. 7c).

As mechanistic target of rapamycin (mTOR) is activated by insulin signalling [37], and may also regulate autophagy [38], we also investigated whether mTOR signalling had any involvement in insulin-induced insulin resistance in podocytes. Inhibition of mTOR with rapamycin had no significant effect on IR levels (Fig. 7d).

Restoring insulin signalling in insulin-resistant human podocytes requires stable expression of both IR and nephrin Human podocytes used in previous studies [15, 39, 40] have become 'naturally' insulin resistant as a consequence
Fig. 6 No significant changes in Ir mRNA in podocytes following chronic insulin exposure. (a) Endpoint RT-PCR and (b) qRTPCR of $I r$ mRNA following chronic exposure of mouse podocytes to insulin; $n=4$. (c) Representative qRT-PCR and (d) endpoint RT-PCR demonstrating the relative abundance of podocyte $I r-A / B$ mRNA following growth in chronic insulin. $* * p<0.01$. Individual changes in (e) $I r-A$ and (f) $I r-B$ mRNA relative to basal condition. No significant differences, unpaired $t$ test; $n=4$ in triplicate a
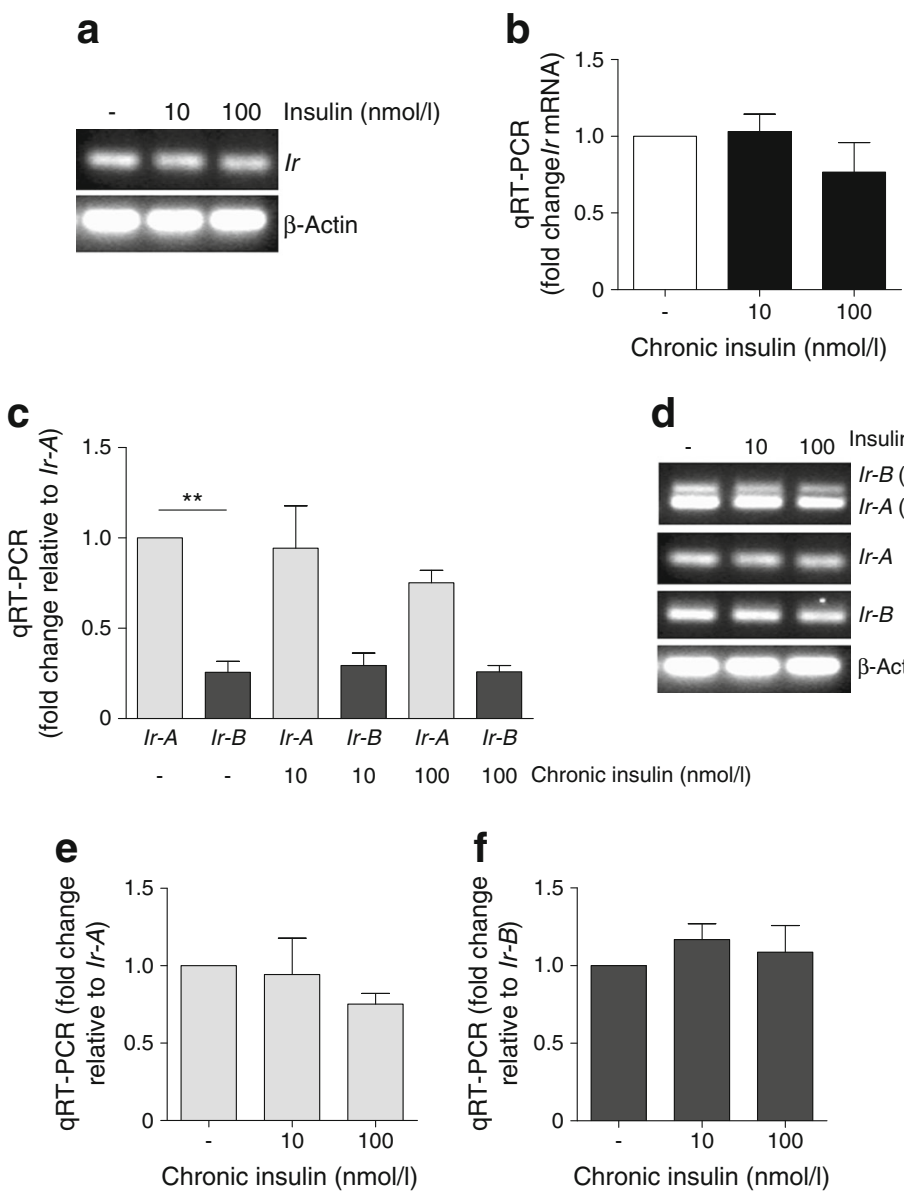

d
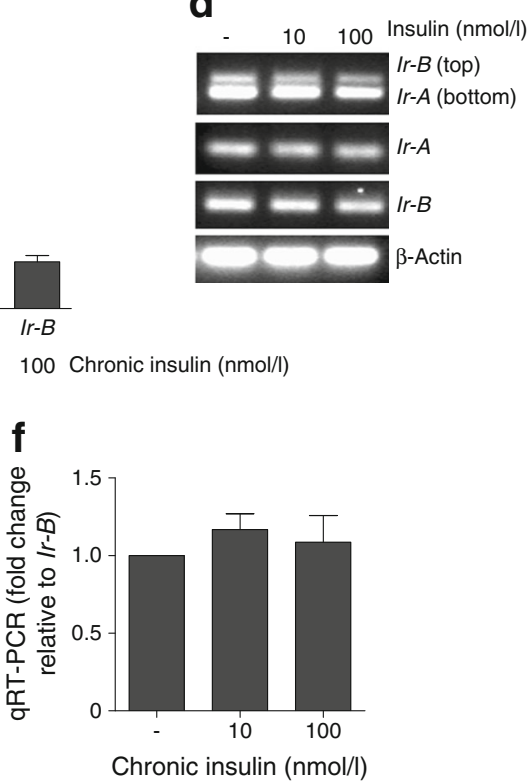
of continuous and historical cell culture conditions, whereby cell culture media were supplemented with insulin at concentrations in excess of $200 \mathrm{nmol} / 1$ [24]. To determine whether IR loss was a primary cause of insulin resistance in these cells, human podocytes (WT) were stably transfected with the human IR using lentivirus (WT-IR podocytes).

In contrast to WT podocytes, there was a significant increase in phosphorylation of Akt following insulin stimulation of WTIR podocytes (Fig. 8a). However, there was no significant increase in the glucose uptake response in either cell line (Fig. 8b).

As we have previously demonstrated that nephrin is necessary for insulin-stimulated glucose uptake in podocytes [39, 40], and nephrin loss is also evident in diabetic environments [41-43], the effect of stable nephrin overproduction in insulin-resistant human podocytes was investigated. As shown in Fig. 8d, podocytes stably producing both IR and nephrin (WT-IR-Neph) retained their insulin-stimulated phosphorylation of Akt. In addition, a significant increase in glucose uptake into WT-IR-Neph podocytes was observed (Fig. 8e). Overproduction of nephrin alone did not rescue insulin-stimulated Akt phosphorylation or glucose uptake (Fig. 8d, f).
As nephrin has a key role in regulating the podocyte actin cytoskeleton [44], and the insulin-stimulated trafficking of glucose transporters (in GLUT storage vesicles) to the plasma membrane relies on the actin cytoskeleton [45], we hypothesised that the requirement of nephrin for glucose uptake in podocytes might also be related to actin modulation. As such, we next investigated insulin-stimulated actin reorganisation in these cells, using automated microscopy and image analysis. Cells positive for actin remodelling were defined as those with a loss of defined (central) F-actin structures, as this is generally considered to indicate reorganisation [46].

Representative images of podocytes stimulated with $100 \mathrm{nmol} / \mathrm{l}$ of insulin for $10 \mathrm{~min}$ are presented in Fig. 9a, which were quantified using an IN Cell Analyzer. In WT-IRNeph podocytes, insulin caused a rapid dose-dependent increase in F-actin remodelling (Fig. 9b). In contrast, insulin stimulation had no effect on actin reorganisation in WT cells or WT cells producing nephrin (WT-Neph), and only a modest effect in WT-IR podocytes (Fig. 9c, e). We also found that the actin remodelling observed in WT-IR-Neph podocytes was completely blocked when these cells were exposed to a diabetic environment (Fig. 9d, e).
Fig. 7 Proteasome and lysosome inhibition blocks insulin-induced IR degradation in podocytes. Podocytes were treated with (a) $50 \mathrm{nmol} / \mathrm{l}$ bafilomycin, (b) $10 \mu \mathrm{mol} / 1 \mathrm{MG} 132$, or (c) both bafilomycin and MG132 for $8 \mathrm{~h}$, alone or in combination with insulin for 8 or $24 \mathrm{~h}$. IR $\beta$ levels were determined by western blotting. Levels of p62 and ubiquitin $(\mathrm{Ub})$ were determined as positive controls for successful lysosomal and proteasomal inhibition, respectively. (d) Representative western blots and matched densitometry showing IR $\beta$ levels after mTOR inhibition ( $24 \mathrm{~h}, 10 \mathrm{nmol} / \mathrm{lrapamycin}$ ) alone or in combination with chronic insulin stimulation; phosphorylation of mTOR (S2448) was determined as a positive control for successful mTOR inhibition; $n=4-6$ experiments. $* p<0.05$, $* * p<0.01$, *** $p<0.001$, oneway ANOVA, Tukey's multiple comparison; ${ }^{\dagger} p=0.0151$, twotailed $t$ test vs $24 \mathrm{~h}$

\section{a}

- - - + + + 8h, $50 \mathrm{nmol} / \mathrm{l}$ bafilomycin - $8 \mathrm{~h} 24 \mathrm{~h}$ - $8 \mathrm{~h} 24 \mathrm{~h} 100 \mathrm{nmol} / \mathrm{l}$ insulin
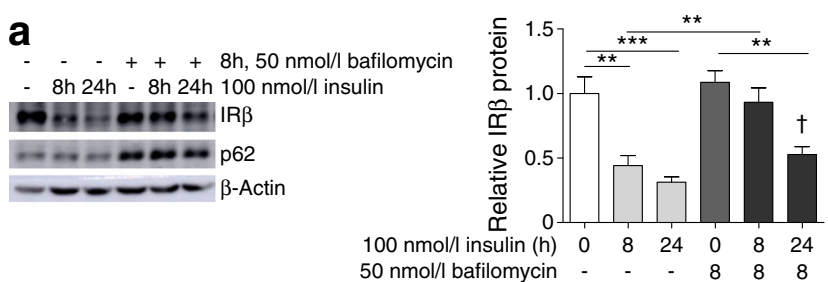

$50 \mathrm{nmol} / \mathrm{l}$ bafilomycin - $\quad-\quad-\quad 8 \quad 8 \quad 8$

b
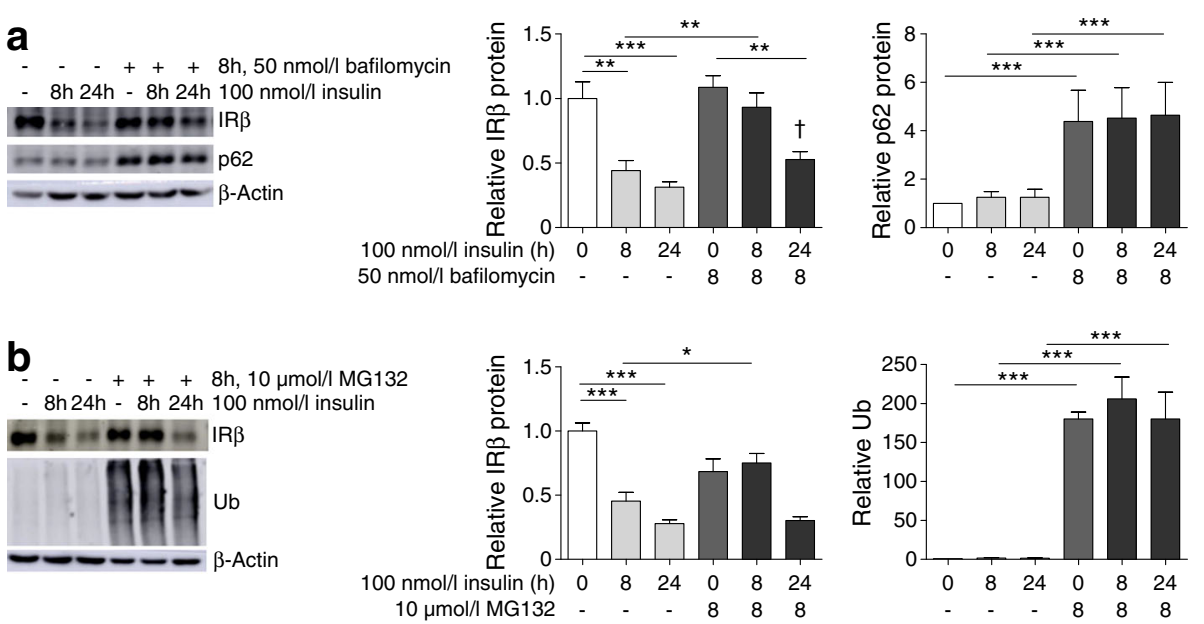

C - - + + + 8h, MG-132 + bafilomycin - $8 \mathrm{~h} 24 \mathrm{~h}-8 \mathrm{~h} 24 \mathrm{~h} 100 \mathrm{nmol} / \mathrm{l}$ insulin

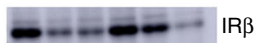

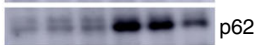
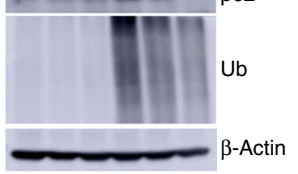

d

- - + + + $24 \mathrm{~h}, 10 \mathrm{nmol} / \mathrm{l}$ rapamycin - $8 \mathrm{~h} 24 \mathrm{~h}$ - $8 \mathrm{~h} 24 \mathrm{~h} 100 \mathrm{nmol} / \mathrm{l}$ insulin

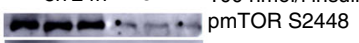

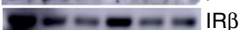
$-0=0-\beta$-Actin

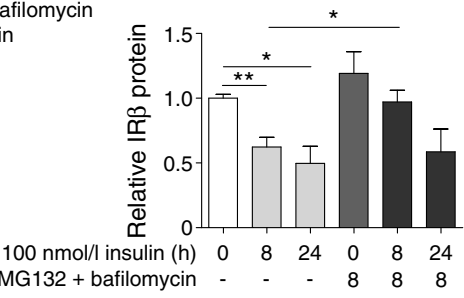

MG132 + bafilomycin - $\quad$ - $\quad$ - 888

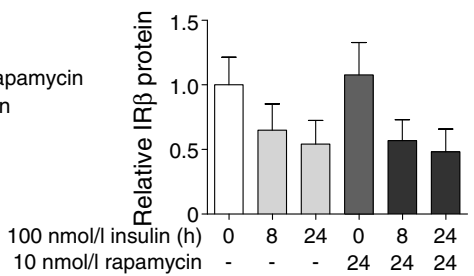


Fig. 8 Stable IR and nephrin expression rescues insulin signalling in insulin-resistant human podocytes. Human (WT) podocytes were stably transfected with lentiviral particles containing human IR (WT-IR), human nephrin (WT-Neph) or both (WTIR-Neph), and insulin responses were investigated. (a)

Representative western blots and matched densitometry demonstrating increased phosphorylation of Akt (S473, T308) in WT-IR podocytes. $* p<0.05, * * p<0.01$,

$* * * p<0.001$, one-way ANOVA,

Tukey's multiple comparison;

$n=4$. Insulin-stimulated glucose uptake experiments showed no significant increase in this response in either (b) WT or (c) WT-IR podocytes. (d)

Representative western blots demonstrating stable expression of nephrin and IR in appropriate cell lines, and insulin-stimulated phosphorylation (nmol/l, $10 \mathrm{~min}$ ) of Akt (S473); $n=3$. (e) WT-IRNeph cells showed a significantly increased percentage glucose uptake following insulin stimulation $(100 \mathrm{nmol} / \mathrm{l})$, compared with unstimulated cells. $* p=0.0142$ MannWhitney vs unstimulated cells. (f) WT-Neph cells have no significant increase in insulinstimulated glucose uptake; $n=4$
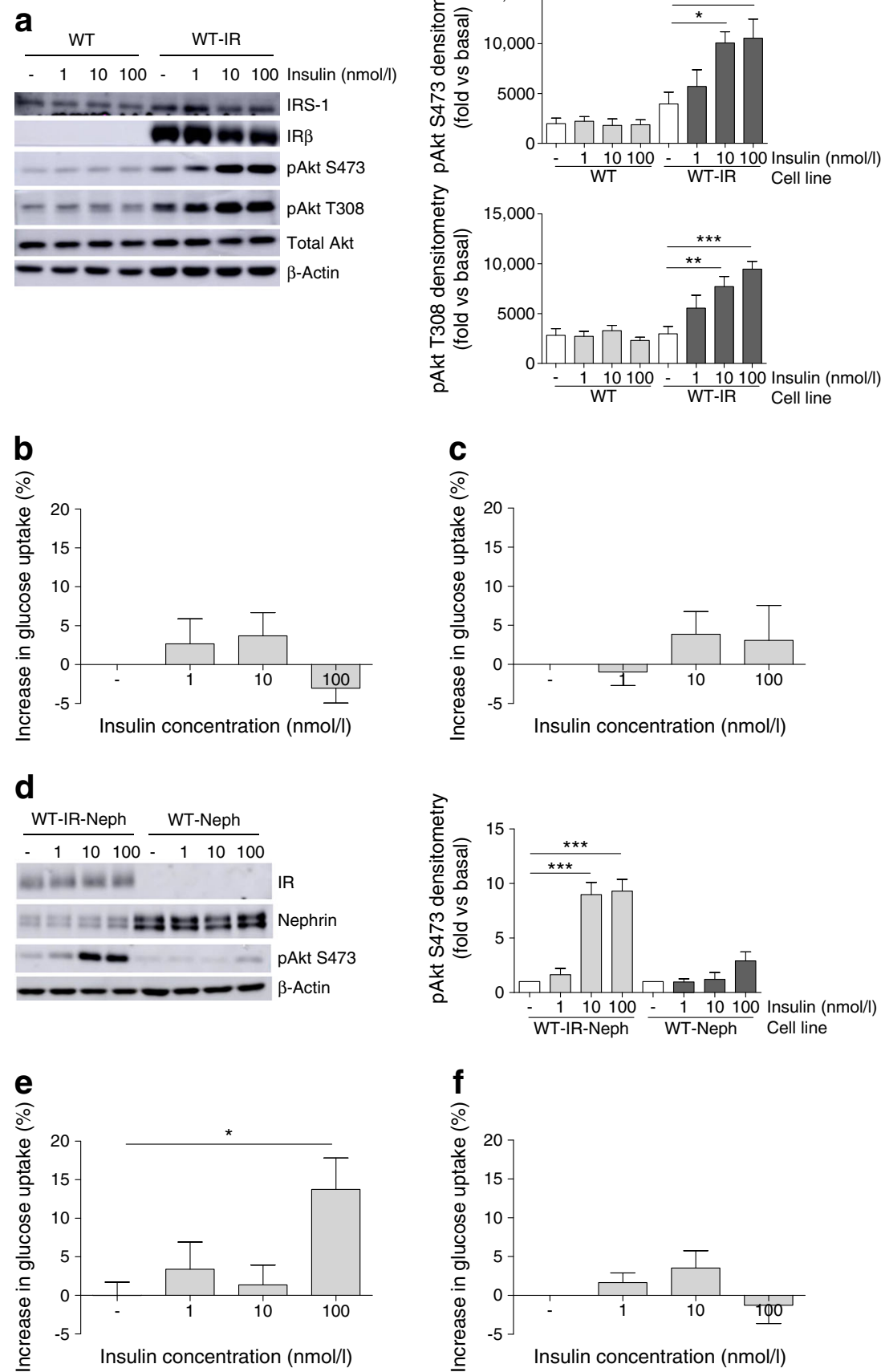
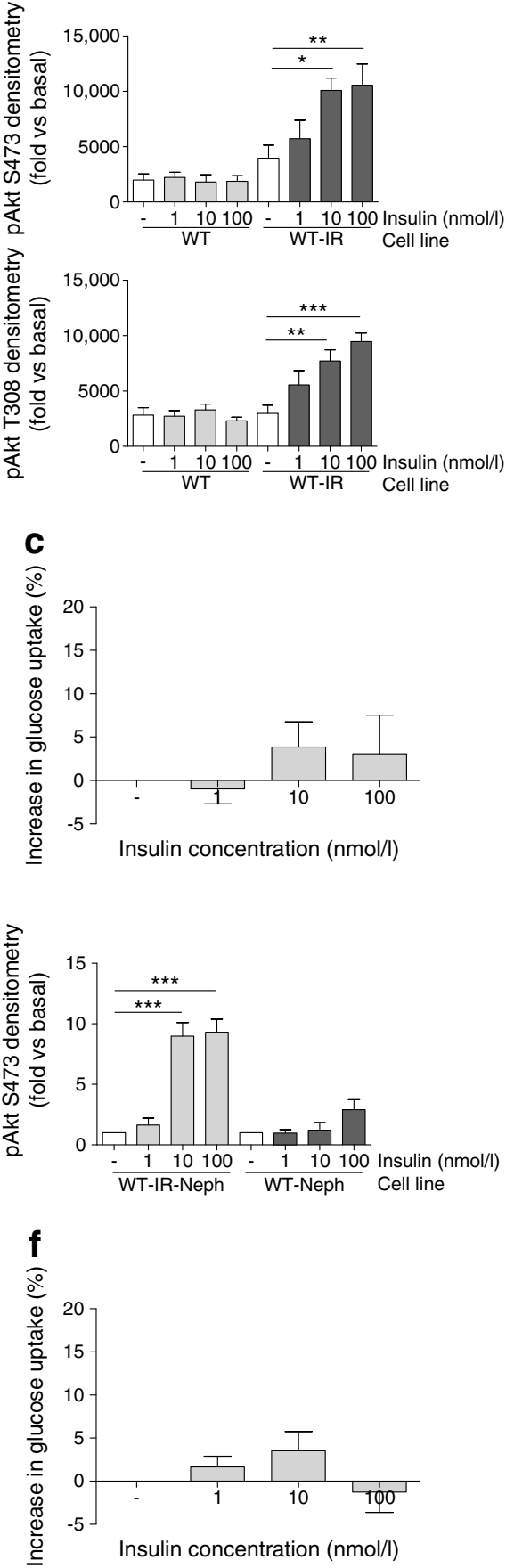

\section{Discussion}

Hyperinsulinaemia is a major metabolic abnormality associated with systemic insulin resistance [47]. In addition to the association between insulin resistance and albuminuria [4-6], hyperinsulinaemia per se is related to albuminuria in type 2 diabetes [48] and in type 1 diabetes, where nephropathy is associated with higher therapeutic doses of insulin [49].

At a cellular level, hyperinsulinaemia may exacerbate insulin resistance, disrupting insulin action at several points within the signalling cascade, in a cell-specific manner [50]. At the level of the IR, chronic insulin stimulation can promote receptor downregulation in classically insulin-responsive tissues such as skeletal muscle and liver [26-28], as well as other cell types including pancreatic alpha cells [25] and cultured neurones [29, 30].

The present study demonstrates, for the first time, that the glomerular podocyte is also subject to insulin-induced IR degradation, resulting in an attenuation of insulin signalling responses. Given that we [16] and others [17] have shown the podocyte-specific knockdown of the IR induces albuminuria and glomerular disease, it is reasonable to predict that this insulin-induced IR loss could further 

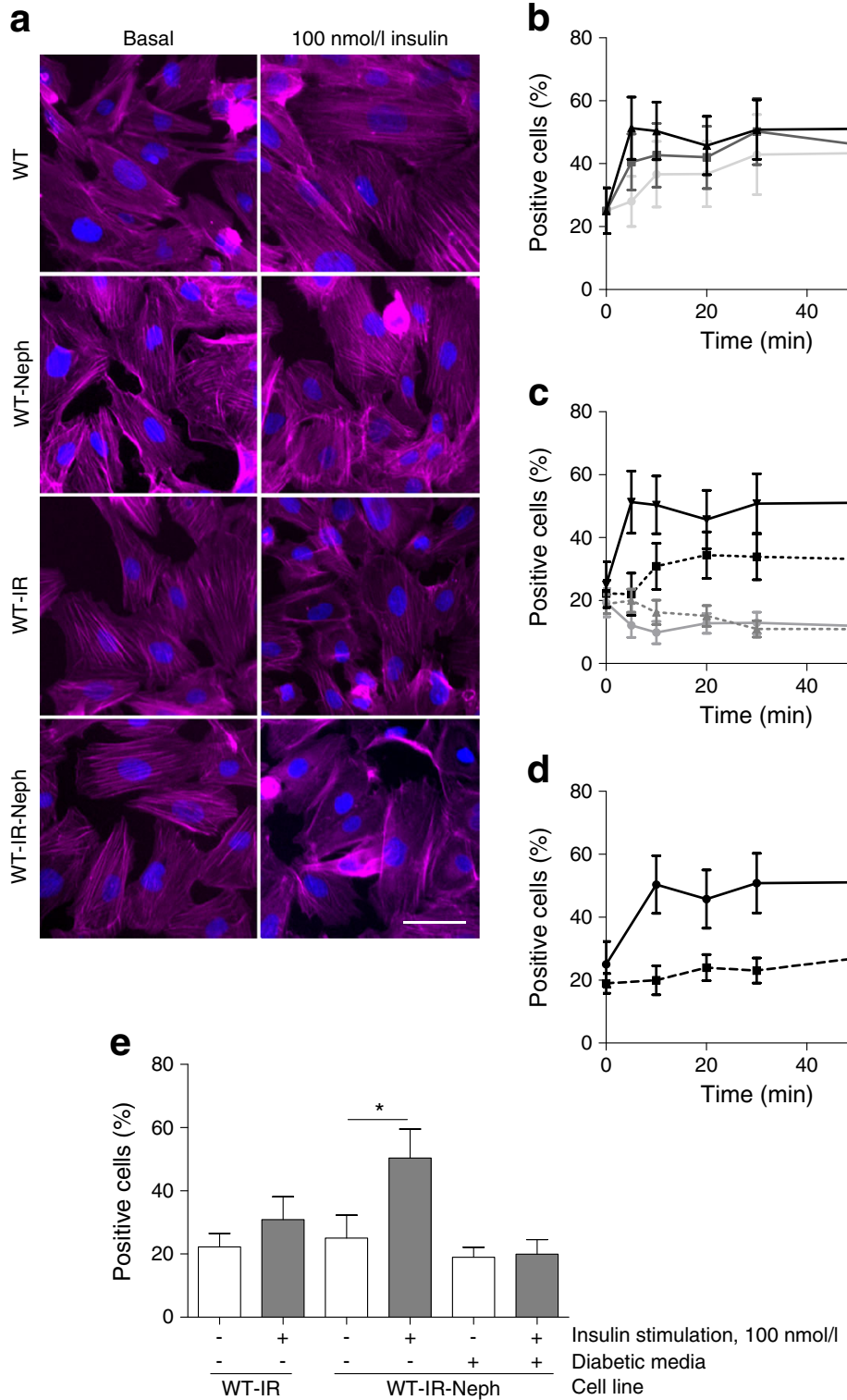

Fig. 9 IR and nephrin expression are both required for effective insulinstimulated actin remodelling in podocytes. Human (WT) podocytes were stably transfected with lentiviral particles containing human nephrin (WT-Neph), human IR (WT-IR) or both (WT-IR-Neph). Cell lines were stimulated with insulin at the stated doses and times, prior to F-actin and nuclear staining. The percentage of cells positive for actin reorganisation was calculated as described in the Methods. Modest changes in brightness and contrast were uniformly applied to all images for visual purposes; unmodified images were used for quantification. (a) Representative fluorescent images of each cell line under basal conditions and following insulin stimulation (100 nmol/1, $10 \mathrm{~min})$. Scale bar, $50 \mu \mathrm{m}$. (b) Percentage of WT-IR-Neph cells displaying evidence of F-actin

contribute to renal disease in settings of diabetes and insulin resistance.

Mechanistically, we demonstrated that insulin-induced IR degradation occurs via a proteasome-dependent and bafilomycin-sensitive pathway. In contrast to studies performed in other cell systems [26, 27], we found that chronic insulin stimulation of podocytes had no significant effect on
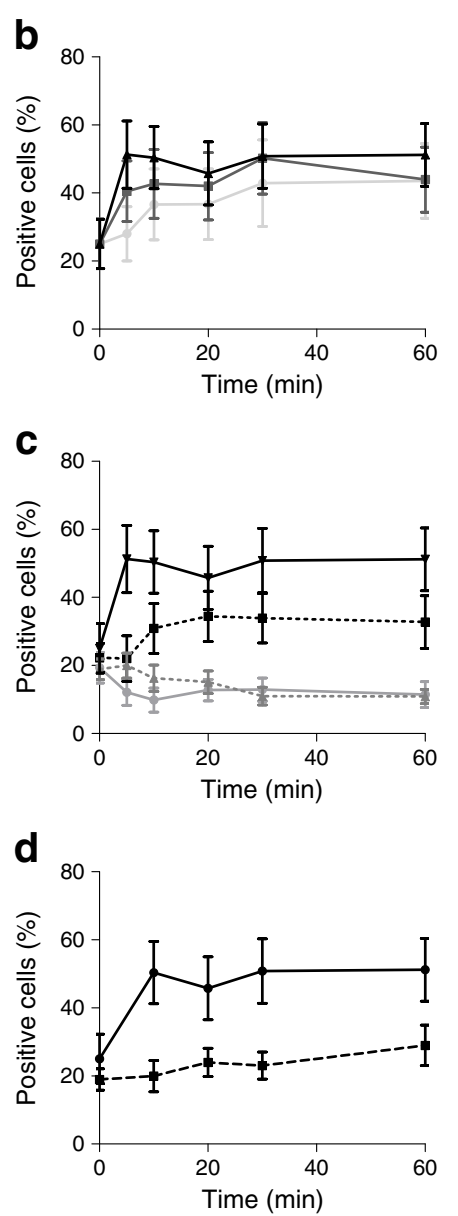

remodelling when stimulated with insulin at $1 \mathrm{nmol} / \mathrm{l}$ (light grey line), $10 \mathrm{nmol} / 1$ (dark grey line) and $100 \mathrm{nmol} / 1$ (black line). (c) Comparison of responses between stable cell lines (grey line, WT; grey dotted line, WTNeph; black dotted line, WT-IR; black line, WT-IR-Neph) stimulated with $100 \mathrm{nmol} / 1$ insulin over the time course. (d) Effect of insulin-resistant conditions on actin remodelling in WT-IR-Neph cells stimulated with $100 \mathrm{nmol} / \mathrm{l}$ insulin over the time course (black line, basal conditions; black dotted line, diabetic media). (e) Bar graph of data from $(\mathbf{c}, \mathbf{d})$ for $100 \mathrm{nmol} / \mathrm{linsulin}$ at $10 \mathrm{~min}$. A significant increase in percentage of WTIR-Neph podocytes positive for actin remodelling was observed; this response was lost in WT-IR-Neph podocytes exposed to insulin-resistant conditions. ${ }^{*} p=0.036$ Mann-Whitney test, $n=3$

IR mRNA. We did, however, demonstrate that the relative abundance of IR-A mRNA is greater than that of IR-B in podocytes. As IR-A is the isoform thought to be responsible for IR-mediated IGF-II signalling, this may be of relevance in IGF-II signalling to this cell $[32,51]$.

Although we found that the insulin-induced degradation of IRS-1 [29] and IRS-2 [52] observed in other cell systems does 
not occur in podocytes, in agreement with previous studies performed in a rat model of streptozotocin-induced diabetes and glomerular endothelial cells [53], we detected a reduction in podocyte IRS-1 content as a consequence of high glucose exposure. Our finding that there was no significant reduction in the insulin-stimulated phosphorylation of Akt or glucose uptake under these conditions is in line with our recent studies suggesting that IRS-2, as opposed to IRS-1, is the principal isoform facilitating podocyte insulin signal transduction to stimulate Akt phosphorylation and glucose uptake [54]. However, this does not eliminate the possibility that IRS-1 could mediate other insulin responses in podocytes, or selectively modulate individual Akt isoforms.

We also demonstrate that while initial insulin signalling events leading to Akt phosphorylation in podocytes do not require nephrin, the stable expression of both IR and nephrin is necessary to mediate insulin-stimulated glucose transport downstream of Akt. Interestingly, it is well documented that nephrin production is also lost early in human diabetic nephropathy [43] and experimental models of diabetes [41, 42].

We hypothesised, given the importance of actin regulation in the insulin-stimulated glucose uptake response [45], that the requirement for nephrin in glucose uptake might also be linked to the role of nephrin in modulating the podocyte actin cytoskeleton [44], a process that is closely related to podocyte function at the filtration barrier. As such, we investigated how insulin-stimulated actin remodelling was affected in our model systems, and found that podocytes with stable over expression of both nephrin and IR were more responsive to insulin in terms of F-actin remodelling, compared with podocytes overexpressing IR alone.

Others have shown nephrin may also interact with the IR [34], investigating whether nephrin also affects localisation of IR, or indeed IR binding to downstream targets, may be of interest. Whether this may also be linked to the ability of nephrin to preferentially phosphorylate selective Akt isoforms remains to be investigated, as Akt2 has been suggested to differentially modulate the podocyte actin cytoskeleton in comparison to Akt1 [55]. In addition, we found that the actin remodelling downstream of insulin was blocked following exposure of these podocytes to a diabetic environment, suggesting that these cells may still become insulin resistant following further challenge with a diabetic environment.

Aside from enhancing cellular glucose uptake and promoting the reorganisation of F-actin structures, podocyte IR signalling has the capacity to regulate vascular endothelial growth factor (VEGF)-A production [56], cell viability [20], reactive oxygen species (ROS) production and autophagy [57], all of which are key in maintaining glomerular filtration and are dysregulated in diabetes. Recently, the importance of

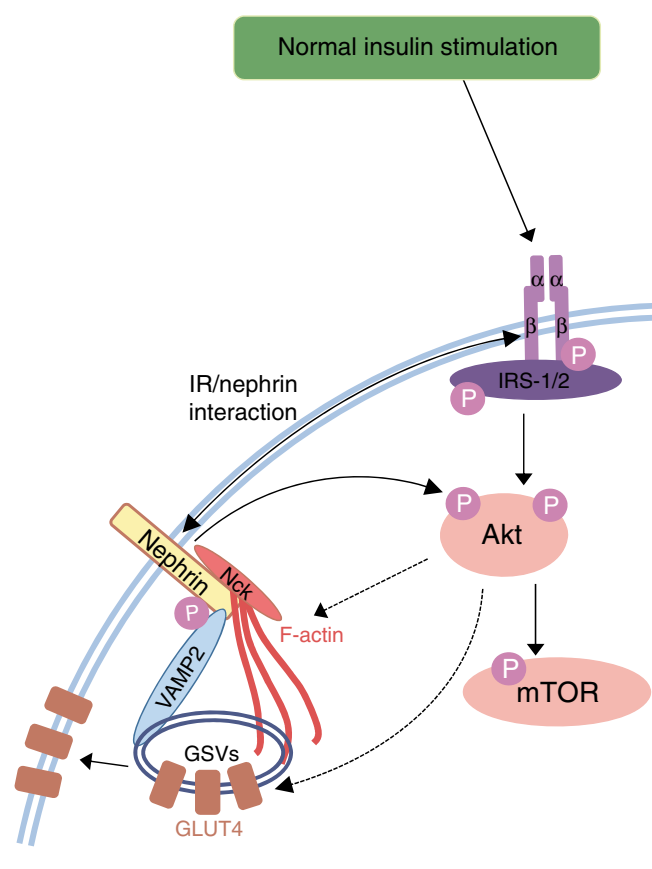

Fig. 10 Proposed mechanism of podocyte IR degradation and its consequences. Normal insulin signalling to podocytes leads to phosphorylation of IRS proteins, predominantly IRS-2 [54], and activation of downstream signalling events, including the phosphorylation of Akt and mTOR. Nephrin is also required for insulin-stimulated glucose uptake (in part via interaction of nephrin with VAMP2 $[39,40]$ and insulin-stimulated actin remodelling). Hyperinsulinaemia, occurring in diabetes and insulin resistance, causes an increase in proteasomal and lysosomal degradation

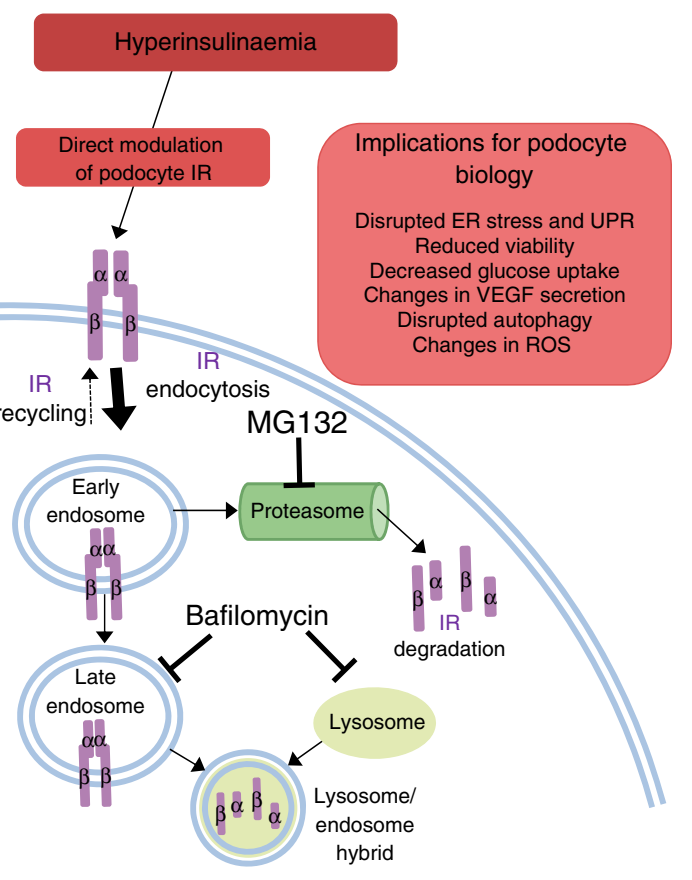

of the podocyte IR, attenuating downstream signal transduction. Ultimately, loss of podocyte IRs may exacerbate albuminuria and features of diabetic nephropathy, dysregulating the ER stress response [17], VEGF-A secretion [56], ROS production [57], autophagy [57], cell viability [20] and actin remodelling, and reducing glucose uptake. ER, endoplasmic reticulum; GSV, GLUT storage vesicle; UPR, unfolded protein response; VAMP2, vesicle-associated membrane protein 2 
IR signalling in the adaptation of podocytes to endoplasmic reticulum stress and the unfolded protein response in diabetes has further highlighted the physiological importance of this pathway in disease responses [17]. A further study has also linked podocyte IR signalling to mitochondrial function, which may be related to the modulation of mTOR signalling [58], which is also crucial in the maintenance of podocytes in health and disease.

As insulin has the capacity to modulate a number of factors vital in podocyte function, we propose that hyperinsulinaemia, via a direct regulation of podocyte IR levels, may directly influence podocyte biology and, subsequently, glomerular function, as depicted in Fig. 10.

In summary, this study demonstrates that podocytes exposed to a diabetic environment become insulin resistant. This response is, at least in part, mediated by chronic exposure to elevated insulin levels promoting podocyte IR degradation. Moreover, we demonstrated that the expression of both IR and nephrin is required for full podocyte insulin sensitivity. Thus, hyperinsulinaemia may further accentuate the progression of renal disease in states of insulin resistance, including diabetic nephropathy, and the maintenance of podocyte nephrin and IR expression and function in these settings may be therapeutically beneficial.

Acknowledgements We thank K. Garner (University of Bristol, UK), who advised on IN Cell data analysis, and L. Farmer (University of Bristol, UK), who assisted with lentivirus work.

Data availability All data generated or analysed during this study are included in this published article (and its supplementary information files). Data sharing is not applicable to this article as no datasets were generated or analysed during the current study.

Funding This study was supported by grants from Kidney Research UK, which funded ACL with a PhD Studentship (ST7/2011) and latterly a project grant to RJMC (RP26/2014), and the MRC, which funds RJMC with a Senior Research Fellowship (MR/K010492/1). RR is supported by a Kidney Research UK John Feehally-Stoneygate award (JR-S/RP/2014/4).

Duality of interest The authors declare that there is no duality of interest associated with this manuscript.

Contribution statement ACL, GIW and RJMC designed the experiments and wrote the manuscript. ACL performed and supervised the experiments. JAH and FB performed and oversaw the animal experiments. VMSB made IR lentivirus and IR-overexpressing cells. LN conditionally immortalised the podocyte cell lines. MVO performed studies on whole mouse glomeruli. LG and GMEP performed initial in vitro studies. HH and RL made the nephrin construct, and RR made the nephrin lentivirus. All authors contributed to the drafting of the manuscript and approved the final version. RJMC is the guarantor of this work.

Open Access This article is distributed under the terms of the Creative Commons Attribution 4.0 International License (http:// creativecommons.org/licenses/by/4.0/), which permits unrestricted use, distribution, and reproduction in any medium, provided you give appropriate credit to the original author(s) and the source, provide a link to the Creative Commons license, and indicate if changes were made.

\section{References}

1. Mogensen CE (1984) Microalbuminuria predicts clinical proteinuria and early mortality in maturity-onset diabetes. N Engl J Med 310:356-360

2. Greenbaum CJ (2002) Insulin resistance in type 1 diabetes. Diabetes Metab Res Rev 18:192-200

3. Reaven GM (1988) Banting lecture 1988. Role of insulin resistance in human disease. Diabetes 37:1595-1607

4. Orchard TJ, Chang YF, Ferrell RE, Petro N, Ellis DE (2002) Nephropathy in type 1 diabetes: a manifestation of insulin resistance and multiple genetic susceptibilities? Further evidence from the Pittsburgh epidemiology of diabetes complication study. Kidney Int 62:963-970

5. Bjornstad P, Snell-Bergeon JK, Rewers M et al (2013) Early diabetic nephropathy: a complication of reduced insulin sensitivity in type 1 diabetes. Diabetes Care 36:3678-3683

6. Parvanova AI, Trevisan R, Iliev IP et al (2006) Insulin resistance and microalbuminuria: a cross-sectional, case-control study of 158 patients with type 2 diabetes and different degrees of urinary albumin excretion. Diabetes 55:1456-1462

7. Pilz S, Rutters F, Nijpels G et al (2014) Insulin sensitivity and albuminuria: the RISC study. Diabetes Care 37:1597-1603

8. Musso C, Javor E, Cochran E, Balow JE, Gorden P (2006) Spectrum of renal diseases associated with extreme forms of insulin resistance. Clin J Am Soc Nephrol: CJASN 1:616-622

9. Kahn SE, Hull RL, Utzschneider KM (2006) Mechanisms linking obesity to insulin resistance and type 2 diabetes. Nature 444:840-846

10. Konner AC, Bruning JC (2012) Selective insulin and leptin resistance in metabolic disorders. Cell Metab 16:144-152

11. Wolf G, Chen S, Ziyadeh FN (2005) From the periphery of the glomerular capillary wall toward the center of disease: podocyte injury comes of age in diabetic nephropathy. Diabetes 54:1626-1634

12. Reddy GR, Kotlyarevska K, Ransom RF, Menon RK (2008) The podocyte and diabetes mellitus: is the podocyte the key to the origins of diabetic nephropathy? Curr Opin Nephrol Hypertens 17:32-36

13. Pagtalunan ME, Miller PL, Jumping-Eagle S et al (1997) Podocyte loss and progressive glomerular injury in type II diabetes. J Clin Invest 99:342-348

14. Toyoda M, Najafian B, Kim Y, Caramori ML, Mauer M (2007) Podocyte detachment and reduced glomerular capillary endothelial fenestration in human type 1 diabetic nephropathy. Diabetes 56: 2155-2160

15. Coward RJ, Welsh GI, Yang J et al (2005) The human glomerular podocyte is a novel target for insulin action. Diabetes 54:3095-3102

16. Welsh GI, Hale LJ, Eremina V et al (2010) Insulin signaling to the glomerular podocyte is critical for normal kidney function. Cell Metab 12:329-340

17. Madhusudhan T, Wang H, Dong W et al (2015) Defective podocyte insulin signalling through p85-XBP1 promotes ATF6-dependent maladaptive ER-stress response in diabetic nephropathy. Nat Commun 6:6496

18. Lay A, Coward RJ (2014) Recent advances in our understanding of insulin signalling to the podocyte. Nephrol Dial Transplant 29: $1127-1133$

19. Lizotte F, Denhez B, Guay A, Gevry N, Cote AM, Geraldes P (2016) Persistent insulin resistance in podocytes caused by epigenetic changes of SHP-1 in diabetes. Diabetes 65:3705-3717 
20. Tejada T, Catanuto P, Ijaz A et al (2008) Failure to phosphorylate AKT in podocytes from mice with early diabetic nephropathy promotes cell death. Kidney Int 73:1385-1393

21. Ostergaard MV, Pinto V, Stevenson K, Worm J, Fink LN, Coward RJ (2016) DBA2J $d b / d b$ mice are susceptible to early albuminuria and glomerulosclerosis that correlates with systemic insulin resistance. Am J Physiol Ren Physiol 312:F312-F321

22. Keir LS, Firth R, May C, Ni L, Welsh GI, Saleem MA (2015) Generating conditionally immortalised podocyte cell lines from wild-type mice. Nephron 129:128-136

23. Ni L, Saleem M, Mathieson PW et al (2012) Nephrology (Carlton, Vic) $17: 525-531$

24. Saleem MA, O'Hare MJ, Reiser J et al (2002) A conditionally immortalized human podocyte cell line demonstrating nephrin and podocin expression. J Am Soc Nephrol 13:630-638

25. Gonzalez M, Boer U, Dickel C et al (2008) Loss of insulin-induced inhibition of glucagon gene transcription in hamster pancreatic islet alpha cells by long-term insulin exposure. Diabetologia 51: 2012-2021

26. Garvey WT, Olefsky JM, Marshall S (1986) Insulin induces progressive insulin resistance in cultured rat adipocytes. Sequential effects at receptor and multiple postreceptor sites. Diabetes 35: 258-267

27. Okabayashi Y, Maddux BA, McDonald AR, Logsdon CD, Williams JA, Goldfine ID (1989) Mechanisms of insulin-induced insulin-receptor downregulation. Decrease of receptor biosynthesis and mRNA levels. Diabetes 38:182-187

28. Gavin JR 3rd, Roth J, Neville DM Jr, de Meyts P, Buell DN (1974) Insulin-dependent regulation of insulin receptor concentrations: a direct demonstration in cell culture. Proc Natl Acad Sci U S A 71: 84-88

29. Mayer CM, Belsham DD (2010) Central insulin signaling is attenuated by long-term insulin exposure via insulin receptor substrate-1 serine phosphorylation, proteasomal degradation, and lysosomal insulin receptor degradation. Endocrinology 151:75-84

30. Kim B, McLean LL, Philip SS, Feldman EL (2011) Hyperinsulinemia induces insulin resistance in dorsal root ganglion neurons. Endocrinology 152:3638-3647

31. Rollason R, Wherlock M, Heath JA, Heesom KJ, Saleem MA, Welsh GI (2016) Disease causing mutations in inverted formin 2 regulate its binding to $\mathrm{G}$-actin, F-actin capping protein (CapZ alpha-1) and profilin 2. Biosci Rep 36:e00302

32. Belfiore A, Frasca F, Pandini G, Sciacca L, Vigneri R (2009) Insulin receptor isoforms and insulin receptor/insulin-like growth factor receptor hybrids in physiology and disease. Endocr Rev 30:586-623

33. Sbraccia P, D'Adamo M, Leonetti F et al (1996) Chronic primary hyperinsulinaemia is associated with altered insulin receptor mRNA splicing in muscle of patients with insulinoma. Diabetologia 39: 220-225

34. Villarreal R, Mitrofanova A, Maiguel D et al (2016) Nephrin contributes to insulin secretion and affects mammalian target of rapamycin signaling independently of insulin receptor. J Am Soc Nephrol: JASN 27:1029-1041

35. Rowzee AM, Ludwig DL, Wood TL (2009) Insulin-like growth factor type 1 receptor and insulin receptor isoform expression and signaling in mammary epithelial cells. Endocrinology 150:3611-3619

36. Rusten TE, Stenmark H (2010) p62, an autophagy hero or culprit? Nat Cell Biol 12:207-209

37. Haar EV, Lee S-I, Bandhakavi S, Griffin TJ, Kim D-H (2007) Insulin signalling to mTOR mediated by the Akt/PKB substrate PRAS40. Nat Cell Biol 9:316-323

38. Jung CH, Ro SH, Cao J, Otto NM, Kim DH (2010) mTOR regulation of autophagy. FEBS Lett 584:1287-1295
39. Coward RJ, Welsh GI, Koziell A et al (2007) Nephrin is critical for the action of insulin on human glomerular podocytes. Diabetes 56: $1127-1135$

40. Lennon R, Welsh GI, Singh A et al (2009) Rosiglitazone enhances glucose uptake in glomerular podocytes using the glucose transporter GLUT1. Diabetologia 52:1944-1952

41. Morito N, Yoh K, Ojima M et al (2014) Overexpression of Mafb in podocytes protects against diabetic nephropathy. J Am Soc Nephrol 25:2546-2557

42. Na J, Sweetwyne Mariya T, Park Ae Seo D, Susztak K, Cagan Ross L (2015) Diet-induced podocyte dysfunction in drosophila and mammals. Cell Rep 12:636-647

43. Doublier S, Salvidio G, Lupia E et al (2003) Nephrin expression is reduced in human diabetic nephropathy: evidence for a distinct role for glycated albumin and angiotensin II. Diabetes 52:1023-1030

44. Jones N, Blasutig IM, Eremina V et al (2006) Nck adaptor proteins link nephrin to the actin cytoskeleton of kidney podocytes. Nature 440:818-823

45. Stöckli J, Fazakerley DJ, James DE (2011) GLUT4 exocytosis. J Cell Sci 124:4147-4159

46. Tojkander S, Gateva G, Lappalainen P (2012) Actin stress fibers-assembly, dynamics and biological roles. J Cell Sci 125(Pt 8):1855-1864

47. Shanik MH, Xu YP, Skrha J, Dankner R, Zick Y, Roth J (2008) Insulin resistance and hyperinsulinemia: is hyperinsulinemia the cart or the horse? Diabetes Care 31:S262-S2S8

48. Catalano C, Muscelli E, Quinones Galvan A et al (1997) Effect of insulin on systemic and renal handling of albumin in nondiabetic and NIDDM subjects. Diabetes 46:868-875

49. Andersen AR, Christiansen JS, Andersen JK, Kreiner S, Deckert T (1983) Diabetic nephropathy in type 1 (insulin-dependent) diabetes: an epidemiological study. Diabetologia 25:496-501

50. Pirola L, Johnston AM, Van Obberghen E (2004) Modulation of insulin action. Diabetologia 47:170-184

51. Hale LJ, Welsh GI, Perks CM et al (2013) Insulin-like growth factor-II is produced by, signals to and is an important survival factor for the mature podocyte in man and mouse. J Pathol 230: 95-106

52. Rui L, Fisher TL, Thomas J, White MF (2001) Regulation of insulin/insulin-like growth factor-1 signaling by proteasomemediated degradation of insulin receptor substrate-2. J Biol Chem 276:40362-40367

53. Mima A, Ohshiro Y, Kitada M et al (2011) Glomerular-specific protein kinase C-beta-induced insulin receptor substrate-1 dysfunction and insulin resistance in rat models of diabetes and obesity. Kidney Int 79:883-896

54. Santamaria B, Marquez E, Lay A et al (1853) IRS2 and PTEN are key molecules in controlling insulin sensitivity in podocytes. Biochim Biophys Acta 2015:3224-3234

55. Canaud G, Bienaime F, Viau A et al (2013) AKT2 is essential to maintain podocyte viability and function during chronic kidney disease. Nat Med 19:1288-1296

56. Hale LJ, Hurcombe J, Lay A et al (2013) Insulin directly stimulates VEGF-A production in the glomerular podocyte. Am J Physiol Ren Physiol 305:F182-F188

57. Audzeyenka I, Rogacka D, Piwkowska A et al (2016) Reactive oxygen species are involved in insulin-dependent regulation of autophagy in primary rat podocytes. Int J Biochem Cell Biol 75:23-33

58. Ising C, Koehler S, Brahler S et al (2015) Inhibition of insulin/IGF-1 receptor signaling protects from mitochondria-mediated kidney failure. EMBO Mol Med 7:275-287 